\title{
Trisomy 18 Case, with and without Cardiac Surgery
}

\author{
Herlina Dimiati ${ }^{1}$, Cut Nanda Feby Ayulinda ${ }^{2}$ \\ ${ }^{1,2}$ Pediatric Cardiology Division, Department of Child Health, Medical School, Universitas Syiah Kuala, Zainoel \\ Abidin Hospital, Banda Aceh, Indonesia \\ herlinadimiati@unsyiah.ac.id
}

\begin{abstract}
Trisomy 18, known as Edwards Syndrome, is the second-largest chromosomal disorder with a poor prognosis with the survival rate from 5 to $10 \%$ at one year of age. The most common factors underlying death are congenital heart defects, heart failure, and pulmonary hypertension. Cardiac surgery performed to patients with Trisomy 18 is associated with a reduction of mortality. This case report presents two cases of Trisomy 18 , in which one of them underwent cardiac surgery.
\end{abstract}

Keywords: trisomy 18; cardiac surgery; mortality

\section{Introduction}

The second most common chromosomal abnormality is Trisomy 18, also known as Edward syndrome, which has a high morbidity and mortality rate.1-3 Trisomy 18 incidence ranges from $1 / 3000$ to $1 / 8000$ cases with a risk of recurrence of cases $<1 \% .1,4-6$ Trisomy 18 is associated with errors in maternal or postzygotic mitosis meiosis, mostly related to function in meiosis II, which causes extra chromosomes (as shown in Figure 1). The relationship waiting for the nondisjunction process is still unknown, thought to be related to maternal age during pregnancy.

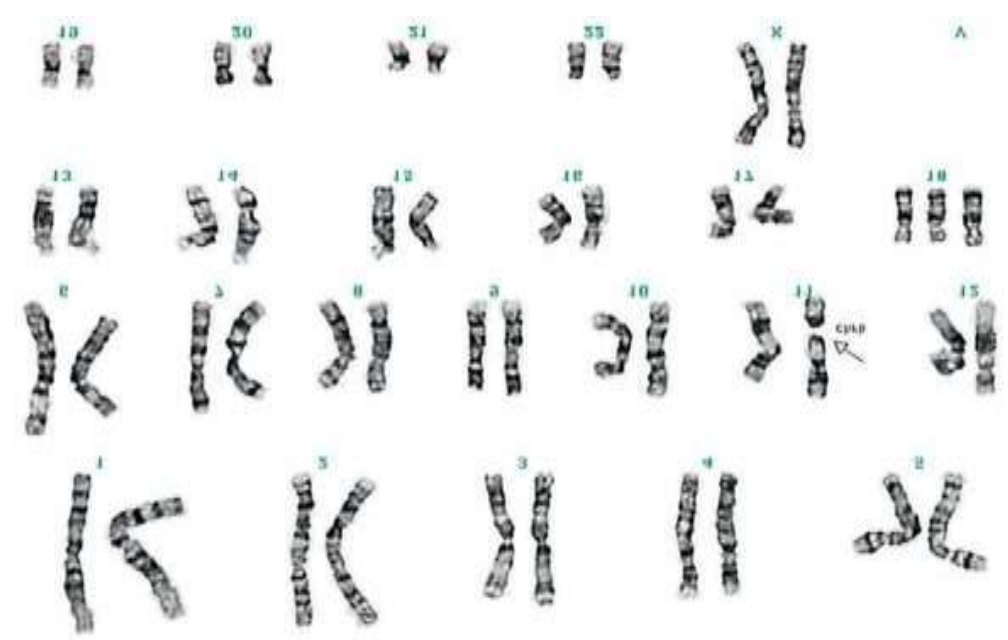

Figure 1. The chromosome of Trisomy 18

Prenatal diagnosis Trisomy 18 can be done by screening maternal age using maternal serum markers (human chorionic gonadotropin, unconjugated estriol, alpha-fetoprotein), and sonography in the second and third trimester of pregnancy. The prenatal sonographic examination will show stunted fetal growth, polyhydramnios, cranial "strawberry-shape", choroid plexus cyst, overlapping of fingers, congenital heart defects, omphalocele, and single umbilical artery. The finding of abnormalities in the late first and second-trimester pregnancy increases the predicted Trisomy 18 rate by $78 \%$. Cesarean section is the recommended delivery when the diagnosis of Trisomy 18 established during pregnancy. 
The clinical features of Trisomy 18 are associated with several anomalies, including abnormal craniofacial (overlapping occiput, narrow bifrontal diameter), low-set and auricular malformations and micrognathia), hand and foot anomalies (clenched hands, overlapping index fingers, and hypoplasia of the nails), low-set and auricular malformations and micrognathia), hand and foot anomalies (clenched hands, overlapping index fingers, and hypoplasia of the nails), low-set and auricular malformations and micrognathia), hand and foot anomalies (clenched hands, overlapping index fingers, and hypoplasia of the nails) chest, inguinal hernia, small pelvis, undescended testis in male infants, and congenital cardiac abnormalities (occurred in almost $90 \%$ of cases, with the common abnormalities including ventricular septal defect (VSD), atrial septal defect (ASD), patent ductus arteriosus (PDA) and polyvalvular disease). In more than $50 \%$ of cases of trisomy 18 , it can be found central nervous system anomalies in the form of cerebellum hypoplasia, corpus callosum agenesis, hydrocephalus, meningomyelocele, and Dandy-walker malformation. Whereas in $10-50 \%$ of cases, it can be found congenital cardiac abnormalities with pulmonary stenosis and aortic coarctation, kidney anomalies such as horseshoe kidney, double ureter, hydronephrosis, and polycystic kidney.1,2,4-9.

The survival rate of Trisomy 18 sufferers in the first year of life is less than $10 \%$, and usually, the life expectancy is not up to one week. The high mortality rate of Trisomy 18 patients is mainly due to Congenital Heart Disease (CHD), and Congestive Heart Failure (CHF), pulmonary hypertension. In some studies, deaths have also been caused by central apnea and failure of multisystem organs.1,2,4,5,7,9,10.

A study suggested surgery performed on a congenital defect in the heart of patient Trisomy 18. Surgical measures performed can reduce pulmonary blood flow, thereby improving the patient's prognosis.1 Several studies mention that heart surgery is associated with a reduction in mortality in Trisomy 18 patients with CHD. The mortality rate in Trisomy 18 patients who did not undergo cardiac surgery was more than $50 \%$, higher than the patients undergoing surgery, which was around $29 \% .8,11$ The longest life span of a Trisomy 18 patient with CHD is 62 months. It is lower than those undergoing surgery, reaching 216 - 241 months of age, 2,6 although it is mentioned that the effective life span of Trisomy 18 patient sufferer is only two to three years after surgery heart.

\section{Cases}

Patient CC: Female, born vaginally, enough term with a birth weight of 1900 grams, from a 26-year-old mother. She was diagnosed with heart disorders in the form of VSD, PDA, and PH at the age of 51 days. VSD closure and PDA ligase closure is performed at 2.5 months without knowing the patient's chromosomal abnormalities. The results of the chromosome analysis come out one day after surgery. During her life, the patient experiences growth disorders and developmental delays. The patient can only lift her head briefly at the age of three years and look for sounds. The patient died at the age of six years and five months due to respiratory problems; she was found suddenly no longer breathing. 


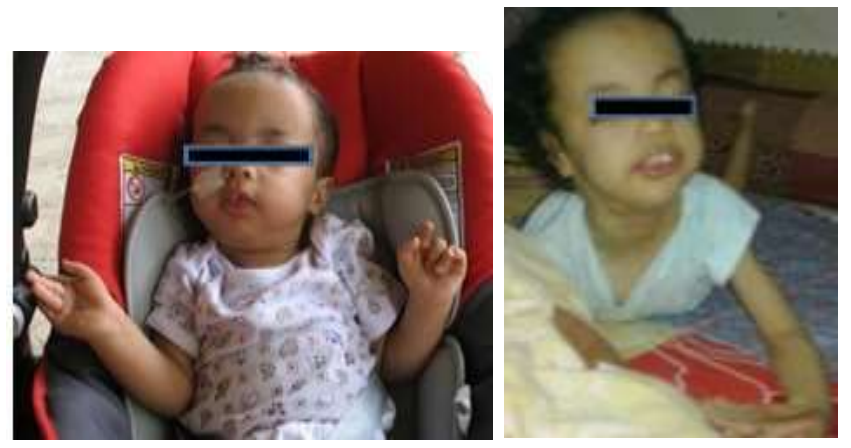

Figure 2. Clinical Pictures of Patient CC

Patient AAS: Female, born vaginally, enough term with a birth weight of 3600 grams, from a 40-year-old mother. She was diagnosed with cardiac abnormalities in the form of VSD, tiny PDA, PFO, and PH. A chromosomal analysis examination was performed when the patient was one year and five months with the results showing trisomy 18 . The patient had never had heart surgery and died at one year and seven months due to respiratory failure on the third day of treatment with severe bronchopneumonia. The patient experienced growth disturbance and developmental delay; she can only lift his head. She was also diagnosed with hypothyroidism, regular controls in the pediatric Endocrine division, and received levothyroxine therapy. The patient suffered from epilepsy and was treated with valproic acid, phenobarbital until Keppra because her seizures persisted every day. The CT scan of the head at the age of 8 months, atrophy of the front temporoparietal lobes left and right, was identified.

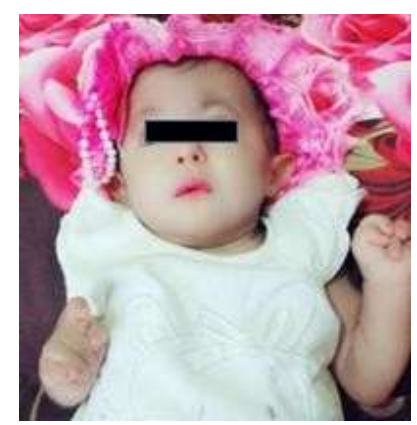

Figure 3. Clinical pictures of patient AAS

\section{Discussion}

Nondisjunction chromosome 18 , or known as Trisomy 18, is more common in girls than boys, in both cases reported were girls. A study found that the risk of trisomy is greater in mothers aged 35 years and over. 4 In these cases, patient AAS was born to an over 35 years mother (42 years old), while patient CC was born to a 26 years old mother.

Both of these patients were with the CHD problem. According to the literature, the most common abnormalities in Trisomy 18 are CHD, and the incidence is up to $90 \%$. Congenital heart defects can be in the form of VSD, PDA, ASD, and polyvalvular disease. As found in both patients, both VSD and PDA are also accompanied by pulmonary hypertension.

In both cases, there are fundamental differences in the CHD management problem. The parents of patient AAS did not agree to the PDA ligation procedure, and she had apnea suddenly due to CHF. Whereas, patient $\mathrm{CC}$ had apnea with a diagnosis of severe aspiration pneumonia. CC did not have CHF. This is consistent with the literature stating that deaths in Trisomy 18 are most frequently caused by CHF and pulmonary hypertension, central apnea, and multisystem organ failure. Aspiration is the most common thing that occurs in Trisomy 
18, leading to recurrent pneumonia. Gastroesophageal reflux is a disorder with a high prevalence, resulting in aspiration.

Gestational age at birth and birth weight increase the life expectancy of trisomy 18 patients after congenital heart surgery. Research by Boghossian et al. found that higher mortality occurred in trisomy 18 patients born with low birth weight.8,12 In contrast to a study conducted by Boghossian, patient CC, who was born at 36 Weeks with birth weight (BW) of 1900 gram, had a longer life span than patient AAS who was born in a full month with BW of 3600 gram. Patient $\mathrm{CC}$ had had a growth disorder since the prenatal period. The literature mentions that the average weight of Trisomy 18 patients at 37 weeks gestation is only around 1700-1800 grams. However, it is not the case with patient AAS. Stunted growth occurred in both patients CC and AAS. This is consistent with previous studies explaining that in patients with Trisomy 18 experience difficulty in feeding due to sucking and swallowing problems.

Patient CC died at the age of six years and five months, while patient AAS died only at one year seven months. Patient CC, who underwent VSD closure and PDA ligation surgery at 2.5 months, had a higher life expectancy than patient AAS. This finding agrees with a study that revealed a higher mortality rate in Trisomy 18 patients who did not undergo cardiac surgery, $>50 \%$ of cases, while it was only about $29 \%$ of cases of those undergoing surgery.8,11 The research by Nakai et al. stated that the life span ranges 115-1800 days for Trisomy 18 patients undergoing heart surgery, while for those undergoing conservative measures, it ranges from 1- 251 days.1 Besides, VSD repair is the most frequently performed procedure for Trisomy patients 18 aged 3-36 months.

Some studies suggested that the life expectancy of people with Trisomy 18 increases with congenital heart surgery, with varying life rates. Some studies explained that the effective time of Trisomy 18 patients was only around 2-3 years after surgery, with a life expectancy around 45\%.3,9,13 Research by Maeda revealed that the life expectancy of postoperative heart patients in Trisomy 18 reached 56\%, with a life span of 2- 216 months, and it was only up to 62 months for unoperated patients, while a study by Muneichi showed that the life span was between 3.3 to 241 months.2,6 The heart surgery was performed to improve pulmonary blood flow, increasing the prognosis of Trisomy 18 patients with CHD.

Delays in cognitive and psychomotor development occurred in both patient $\mathrm{CC}$ and AAS, even though patient $\mathrm{CC}$ had a longer life span. This is consistent with the literature explaining that severe psychomotor development delays occur in people with Trisomy 18 with a long life span.

\section{Conclusion}

Heart surgery can increase life expectancy in Trisomy 18 patients with a CHD. However, the indications of heart surgery should be performed based on the doctor's decision, and the family must be given enough information to be able to participate in the decision-making process.

\section{References}

Bawo D. D. S, Ayodele, A., Oyedeji, Solomon, P. B., Frankland, O., Briyai, Jasper, F. N., and Abowei. 2020. Assessment of the Larvicidal Efficacy of the Hexane-Leaf-Extracts of Selected Tropical Plant Species. Budapest International Research in Exact Sciences (BirEx) Journal (2): 136-140.

Boghossian NS, Hansen NI, Bell EF, Stoll BJ, Murray JC, Carey JC, Adams-Chapman I, Shankaran S, Walsh MC, Laptook AR, Faix RG. Mortality and morbidity of VLBW 
infants with trisomy 13 or trisomy 18. Pediatrics. 2014 Feb 1;133(2):226-35.

Bruns D. A, Martinez A. An analysis of cardiac defects and surgical interventions in 84 cases with full trisomy 18. American journal of medical genetics Part A. 2016 Feb;170(2):337-43.

Bruns D, Campbell E. Twenty-two survivors over the age of 1 year with full trisomy 18: presenting and current medical conditions. American Journal of Medical Genetics Part A. 2014 Mar;164(3):610-9.

Cereda A, Carey JC. The trisomy 18 syndrome. Orphanet journal of rare diseases. 2012 Dec $1 ; 7(1): 81$.

Costello JP, Weiderhold A, Louis C, Shaughnessy C, Peer SM, Zurakowski D, Jonas RA, Nath DS. A contemporary, single-institutional experience of surgical versus expectant management of congenital heart disease in trisomy 13 and 18 patients. Pediatric cardiology. 2015 Jun 1;36(5):987-92.

Halawa, B., Azwana, and Panggabean, E. L. 2019. Sensitivity of Larva Spodoptera litura Against the Density of Spores of Fungi Metarhizium anisopliae on the Onion Plant Red (Allium cepa) in the Laboratory. Budapest International Research in Exact Sciences (BirEx) Journal (1): 35-41.

Imataka $\mathrm{G}$, Suzumura $\mathrm{H}$, Arisaka O. Clinical features and survival in individuals with trisomy 18: A retrospective one-center study of 44 patients who received intensive care treatments. Molecular medicine reports. 2016 Mar 1;13(3):2457-66.

Kaneko Y, Kobayashi J, Yamamoto Y, Yoda H, Kanetaka Y, Nakajima Y, Endo D, Tsuchiya $\mathrm{K}$, Sato H, Kawakami T. Intensive cardiac management in patients with trisomy 13 or trisomy 18. American journal of medical genetics Part A. 2008 Jun 1;146(11):1372- 80.

Kosiv KA, Gossett JM, Bai S, Collins RT. Congenital heart surgery on in-hospital mortality in trisomy 13 and 18. Pediatrics. 2017 Nov 1;140(5):e20170772.

Maeda J, Yamagishi H, Furutani Y, Kamisago M, Waragai T, Oana S, Kajino H, Matsuura H, Mori K, Matsuoka R, Nakanishi T. The impact of cardiac surgery in patients with trisomy 18 and trisomy 13 in Japan. American journal of medical genetics Part A. 2011 Nov;155(11):2641-6.

Mullin J, Wolfe J, Bluebond-Langner M, Craig F. Experiences of children with trisomy 18 referred to pediatric palliative care services on two continents. American Journal of Medical Genetics Part A. 2019 Jun;179(6):903-7.

Muneuchi J, Yamamoto J, Takahashi Y, Watanabe M, Yuge T, Ohno T, Imoto Y, Sese A, Joo K. Outcomes of cardiac surgery in trisomy 18 patients. Cardiology in the Young. 2011 Apr;21(2):209-15.

Nakai Y, Asano M, Nomura N, Matsumae H, Mishima A. Effectiveness of cardiac surgery in patients with trisomy 18: a single-institutional experience. Cardiology in the Young. 2016 Oct;26(7):1391-6.

Syahrul, Mutiawati, E., Astini, N., Fajri, N., and Suherman. 2020. Clinical Characteristic Myasthenia Gravis among Indonesians. Budapest International Research in Exact Sciences (BirEx) Journal (2): 257-263.

Yamagishi H .Cardiovascular Surgery for Congenital Heart Disease Associated with Trisomy Gen Thorac Cardiovasc Surg 2010 May;58:217-219. 